\title{
Effect of Lubrication on Friction and Wear Properties of PEEK with Steel Counterparts
}

\author{
Go Tatsumi $^{1,2)^{*}}$, Monica Ratoi ${ }^{11}$, Yuji Shitara ${ }^{2)}$, Kiyomi Sakamoto ${ }^{2)}$ and Brian G. Mellor ${ }^{3)}$ \\ ${ }^{1)}$ National Centre for Advanced Tribology at Southampton (nCATS), University of Southampton, \\ Highfield, Southampton SO17 1BJ, United Kingdom \\ ${ }^{2)}$ Lubricant R\&D Dept., JXTG Nippon Oil \& Energy Corporation, \\ 8 Chidoricho, Naka-ku, Yokohama, Kanagawa 231-0815, Japan \\ ${ }^{3)}$ Faculty of Engineering and Physical Sciences, University of Southampton, \\ Highfield, Southampton SO17 1BJ, United Kingdom \\ *Corresponding author: Go Tatsumi (tatsumi.go@jxtg.com)
}

Manuscript received 29 June 2019; accepted 22 August 2019; published 15 December 2019

Presented at the International Tribology Conference Sendai 2019, 17-21 September, 2019

\begin{abstract}
Poly-Ether-Ether-Ketone (PEEK) has been widely used for tribological applications with steel counterparts because of its superior mechanical and self-lubricating properties. Fluid lubrication has the potential to further improve the performance of PEEK/steel contacts but its effect has been shown to depend on the operating conditions. To elucidate these aspects and establish the mechanism of fluid lubrication, the friction and wear properties of the PEEK/steel contacts in both dry and poly- $\alpha$-olefin (PAO) lubricated conditions were investigated by tribological tests and surface analytical techniques. The nanoindentation measurements showed that lubrication with PAO had a softening effect on the wear track of PEEK, but no correlation was established with the wear of PEEK. The tribological behavior, for both dry and lubricated contacts, was correlated with the thickness of PEEK transfer films on the steel counterparts. The thickness of these films, as determined by Electron Probe Micro Analysis (EPMA) and X-ray Photoelectron Spectroscopy (XPS), was controlled by the rates of their formation and removal and depended on the operating conditions. Moreover, lubrication with PAO inhibited not only the formation but also the removal of PEEK transfer films. This study thus sheds light on the mechanism of fluid lubrication of the PEEK/steel contacts.
\end{abstract}

\section{Keywords}

lubrication, poly-ether-ether-ketone (PEEK), polymers, friction, wear, transfer films, mini traction machine (MTM)

\section{Introduction}

Polymers and polymer-based composites are becoming preferred materials in many tribological applications. Compared with metals, polymer materials have advantages such as lightweight, reduced noise and self-lubricating properties which make their use in automotive, aerospace, medical, industrial applications highly desirable [1-9]. On the other hand, the mechanical strength and thermal stability of polymers are lower than those of metals, and therefore they tend to suffer from failures such as wear, local melting and pitting when used in severe conditions [10-13].

Among the many types of polymers, Poly-Ether-EtherKetone (PEEK) has suitable properties for tribological applications under severe conditions due to its superior mechanical properties and higher thermal stability than those of other conventional polymers $[1,3,14]$. PEEK is commonly used with steel counterparts, and numerous studies have investigated the friction and wear properties of PEEK/steel contacts in dry conditions [15-21]. One of the key factors of the favorable tribological properties in dry conditions is the formation of transfer films of PEEK on steel counterparts [19, 22]. They act as protective films avoiding the direct contact of PEEK with hard asperities of steel and thus reduce friction and wear. In-situ observation of the PEEK wear process was reported by Puhan and Wong [23] and suggested that PEEK wear debris ploughed by the asperities of the counter surface re-entered the contact and became trapped between asperities of the counter surfaces by compression, forming PEEK transfer films on the counter surfaces. Kalin et al. [24] reported transfer-film thickness and coverage showed a good correlation with the friction and wear performances of PEEK and PEEK composites.

While there are many attempts to improve the tribological performance of PEEK from the material side, as in the case of PEEK composites with specific fillers [24-29], fluid lubrication has the potential to further reduce friction and wear of PEEK. Zhang 
et al. [30] reported lubrication with diesel fuel decreased friction and wear of PEEK in a PEEK plate/steel ring sliding contact while observing the transfer film on steel counterparts. However, compared to dry conditions, little work has been reported on the tribological performance of PEEK in fluid lubricated contacts and most of it was focused on water lubrication [31,32]. Additionally, Kurdi et al. [33] showed that water lubrication of a steel/ PEEK contact reduced friction, but increased the wear of PEEK dramatically compared with dry conditions. As a PEEK transfer film on the steel counterpart was not observed in water lubricated conditions, the authors concluded that water lubrication inhibits the formation of stable transfer films on steel counterparts. Yamamoto and Takashima [34] reported a similar acceleration of wear of PEEK in water lubrication. Additionally, the hardness of the PEEK sliding surface decreased in water lubrication, while the mere immersion in water did not lead to any changes. The authors postulated that the softening of the rubbing surface during water lubrication may be due to the interaction between water molecules and the carbonyl group in the PEEK molecular structure and was considered to be the key factor for the high wear of PEEK. Similar softening of PEEK in water lubricated conditions were reported by Yamaguchi and Hokkirigawa [35]. Some work on oil lubricated PEEK was also reported [36-38], but the key factors mentioned above were not fully investigated and the reason why fluid lubrication can have positive or negative effects on wear of PEEK depending on tribological conditions remains unclear.

The current study aimed to investigate the effect of fluid lubrication on tribological properties of PEEK and elucidate its mechanism of lubrication. The friction and wear behavior of PEEK was studied under dry and oil lubricated conditions in sliding-rolling contact with steel counterparts. Most of the previously reported work was carried out in pure sliding contacts. However, lubricated PEEK/steel contacts are envisaged in tribological applications such as gears and bearings that experience sliding-rolling contacts. The two most important factors influencing tribological properties, the PEEK transfer films on steel counterparts and modifications of PEEK hardness after tribological testing have been carefully investigated.

A better understanding of the effect and working mechanism of fluid lubrication is essential to formulating optimal lubricants and developing more suitable systems for PEEK applications.

\section{Experimental methods}

\subsection{Materials}

PEEK plate specimens $40 \times 40 \times 2 \mathrm{~mm}$ were injection molded from commercially available PEEK (Solvay ${ }^{\circledR}$ KT-820 NT). The Ra surface roughness of the PEEK plates was less than $0.05 \mu \mathrm{m}$ and they were used without polishing. To evaluate the effect of lubrication on the tribological properties of PEEK in the boundary lubrication regime, half-inch steel balls (AISI 52100) were purchased from PCS instruments Ltd and shot blasted to $R a$ of approximately $0.5 \mu \mathrm{m}$. New plates and balls were used for each test and cleaned with a hydrocarbon-mix solvent (FASTCLEAN 201, CRC Industries UK Ltd) and isopropanol prior to the test. A poly- $\alpha$-olefin (PAO) oil with a density of 0.819 $\mathrm{g} / \mathrm{cm}^{3}$ and viscosity of $17.5 \mathrm{cSt}$ at $40^{\circ} \mathrm{C}$ and $3.9 \mathrm{cSt}$ at $100^{\circ} \mathrm{C}(\sim 31$ cSt at $25^{\circ} \mathrm{C}$ ) was used as lubricant.

\subsection{Tribological tests}

Tribological tests were carried out using a Mini Traction
Machine (MTM) from PCS instruments Ltd in dry and PAO lubricated conditions. The PEEK plate was placed on top of a bespoke MTM disc and secured in place with the nut (Fig. 1). The test conditions are summarized in Table 1. The steel ball and disc were driven independently to create a mixed slidingrolling contact with slide-roll ratios (SRRs) of 50\% and 100\%. SRR is defined as the ratio of sliding speed to entrainment speed (in pure sliding SRR is $200 \%$ ). All tests were carried out under an entrainment speed of $0.5 \mathrm{~m} / \mathrm{s}$ and an applied load of $50 \mathrm{~N}$ for 60 minutes at ambient temperature (approximately $25^{\circ} \mathrm{C}$ ). The maximum Hertzian contact pressure $\left(\mathrm{P}_{\max }\right)$ was calculated as 0.16 GPa using an elastic modulus of 3.83 GPa and Poisson's ratio of 0.33 for PEEK (adapted from supplier's catalogue). The wear tracks on the PEEK plates were analyzed at the end of the tribological tests to quantify wear and changes in hardness. The wear rates of PEEK plates were calculated from wear profiles measured with a stylus profilometer (Talysurf, Taylor Hobson).

\subsection{Nanoindentation measurements}

Nanoindentation measurements were performed on an iMicro nanoindenter (NANOMECHANICS, Inc.) equipped with a Berkovich tip. To evaluate the hardness of PEEK as a function of indentation depth, the continuous stiffness measurement (CSM) technique [39-41] was employed by applying a small, sinusoidally varying signal on top of a DC signal driving the indenter. The hardness at each indentation depth is determined by analyzing the response of amplitude and phase. The frequency and displacement amplitude values were $110 \mathrm{~Hz}$ and $1 \mathrm{~nm}$, respectively. Under a load range of $50 \mathrm{mN}$, a target depth of $3 \mu \mathrm{m}$ and a strain rate of $0.01 \mathrm{~s}^{-1}$, sixteen points at 50 $\mu \mathrm{m}$ intervals were measured inside and outside of wear tracks for each PEEK plate. The average hardness at each indentation depth and the standard deviation were then calculated. a)

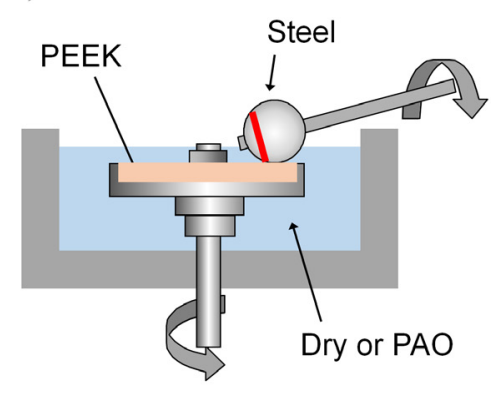

b)

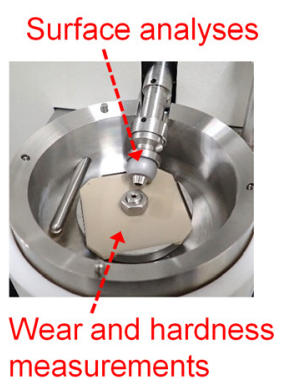

Fig. 1 MTM (a) schematic configuration and (b) set-up appearance

Table 1 Tribological test conditions

\begin{tabular}{|c|c|c|c|c|}
\hline Test condition & \multicolumn{2}{|c|}{ Dry } & \multicolumn{2}{|c|}{$\mathrm{PAO}$} \\
\hline SRR & $50 \%$ & $100 \%$ & $50 \%$ & $100 \%$ \\
\hline Entrainment speed & \multicolumn{4}{|c|}{$0.5 \mathrm{~m} / \mathrm{s}$} \\
\hline Load / $\mathrm{P}_{\max }$ & \multicolumn{4}{|c|}{$50 \mathrm{~N} / 0.16 \mathrm{GPa}$} \\
\hline Temperature & \multicolumn{4}{|c|}{ Ambient $\left(\sim 25^{\circ} \mathrm{C}\right)$} \\
\hline Testing time & \multicolumn{4}{|c|}{60 minutes } \\
\hline Lambda ratio & \multicolumn{2}{|c|}{ - } & \multicolumn{2}{|c|}{$\sim 0.2$} \\
\hline
\end{tabular}


2.4 Surface analyses (EPMA, XPS)

Electron Probe Micro Analysis (EPMA) and X-ray Photoelectron Spectroscopy (XPS) were carried out on the transfer films found on steel balls after tribological testing using a JXA-8530F (JEOL, Ltd.) and a PHI 5000 VersaProbe III (UlvacPhi, Inc.). Test specimens were rinsed with hydrocarbon solvent and dried before measurement. The EPMA carbon maps were acquired with a $15-\mathrm{kV}$ beam at $100 \mathrm{nA}$ current. The XPS spectra were obtained using a monochromatic $\mathrm{A} 1 \mathrm{~K} \alpha$ source. The $\mathrm{C}$ $1 \mathrm{~s}$ detailed scan was performed with a spot size of $100-\mu \mathrm{m}$ diameter. In general, EPMA shows average surface elemental composition up to a few $\mu \mathrm{m}$ depth while XPS reflects the nature on the uppermost surfaces up to a few nm depth [42]. This makes both techniques suitable for investigation of thickness and chemical nature of transfer films.

\section{Results and discussion}

\subsection{Tribological tests}

Friction coefficient values as a function of time and the averaged friction during the last ten minutes of testing for the dry and lubricated tests are illustrated in Figs. 2 and 3. Regardless of SRR, a significant friction reduction was achieved in the PAO lubricated tests compared to the dry ones. Unexpectedly, for both dry and lubricated contacts, friction was lower for $100 \%$ SRR than for $50 \%$ SRR.

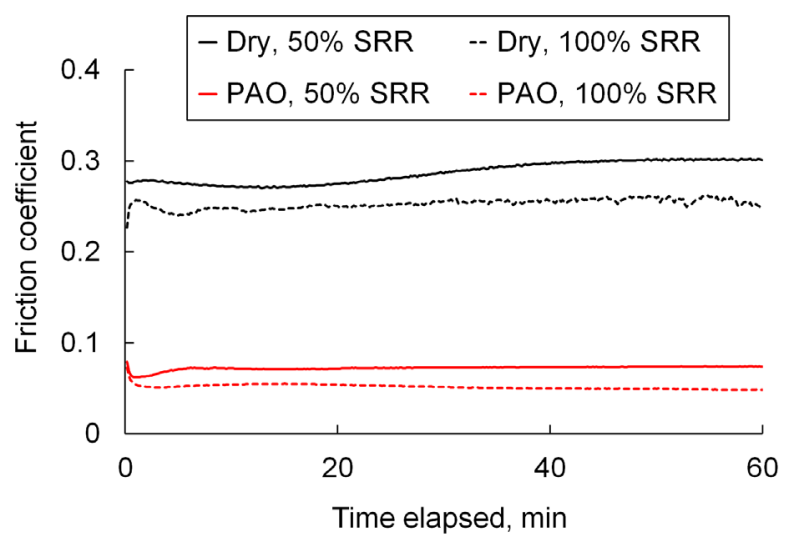

Fig. 2 Friction coefficient as a function of test time

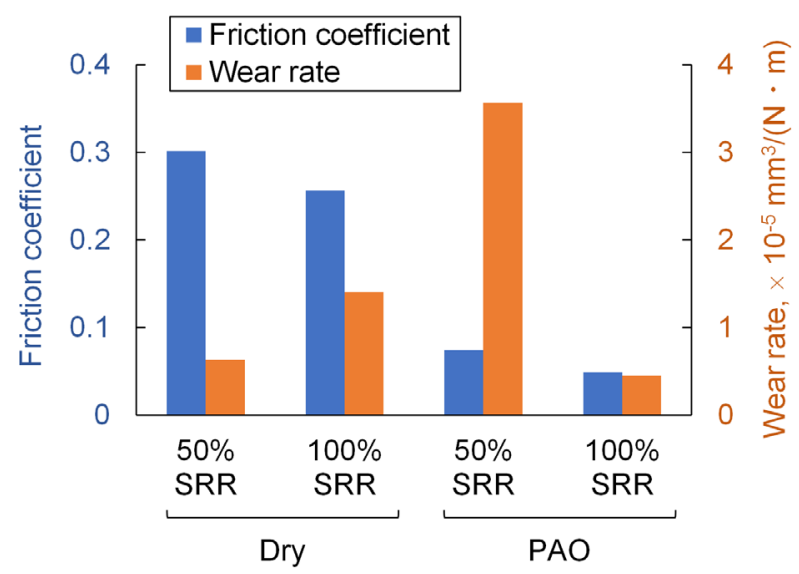

Fig. 3 Friction coefficient averaged during the last ten minutes and wear rates of PEEK plates
The wear rates of PEEK plates in Fig. 3 show a different trend than friction coefficients. In dry conditions, the 100\% SRR wear rate was higher than at $50 \%$ despite showing the lower friction. In the case of lubricated contacts, the wear trend was opposite the unlubricated ones, with $100 \%$ SRR resulting in lower wear than $50 \%$ SRR. Additionally, while lubrication exerted a negative effect on wear at $50 \%$ SRR it had a positive one at $100 \%$ SRR, and almost no correlation was seen between the friction and wear results.

Theoretically, the different values of SRR used for testing have no effect on the lubrication regime. The lubricated contacts at both SRRs (50\% and 100\%) experienced the same entrainment speeds $(0.5 \mathrm{~m} / \mathrm{s})$ and therefore similar lubricant film thicknesses and Lambda ratios. The testing conditions employed in this study were borderline between piezoviscous-elastic and isoviscous-elastic lubrication. Therefore, the oil film thickness calculations give almost the same results irrespective of using the equations for piezoviscous-elastic [43] or isoviscous-elastic lubrication $[44,45]$. The calculated values of approximately 0.2 for Lambda ratios entail that both PAO 50\% and 100\% tests operated in the boundary lubrication regime.

To investigate the wear mechanism of PEEK plates, optical images of the wear tracks under two magnifications were analyzed (Fig. 4(a-h)). The high magnification images show that the appearance of the wear tracks is different, especially in the case of two specimens: the dry $50 \%$ SRR and PAO 50\% SRR. The wear track on the dry $50 \%$ SRR plate showed signs of adhesive or fatigue wear. By contrast, the wear track of the PAO 50\% SRR showed the characteristic pattern of abrasion as observed by Schallamach for abrasive wear of rubber [46]. When tests were conducted with smooth steel balls and thus in the hydrodynamic lubrication regime, there was almost no wear on the PEEK plates regardless of SRR. Further aspects of the wear mechanisms will continue to be investigated in the following sections.

Previous research pointed out that lubrication can have an important effect on the hardness of PEEK and the formation of transfer films on steel counterparts. The current study decided to study the mechanism of lubrication of PEEK/steel contacts and thus understand their friction and wear behavior by focusing on these two key factors.

\subsection{Nanoindentation investigations of PEEK hardness}

Changes in PEEK hardness after testing were measured using nanoindentation. Hardness measurements were carried out on PEEK plates outside and inside of wear tracks and the hardness values were plotted as a function of indentation depth (Fig. 4(i1)). In all tests, hardness showed high values at indentation depth values $<0.5 \mu \mathrm{m}$. These values are thought to be attributed to a phenomenon known as indentation size effect (ISE), and not regarded as physically significant because they are distorted by the inadequacies in the procedures applied to provide corrections for the imperfections in the tip geometry $[41,47,48]$.

The hardness at $2 \mu \mathrm{m}$ indentation depth for the four specimens are graphically summarized in Fig. 5 . The hardness values for the specimens used in the dry $50 \%$ and $100 \%$ SRR tests are very similar inside and outside of wear track. By contrast, the PAO lubricated specimens show a significant decrease in hardness (of approximately 23\%) inside the wear tracks for both $50 \%$ and $100 \%$ SRR tests. This implies that lubrication with PAO reduced the hardness of the PEEK plates, regardless of SRR. Interestingly, lubrication with PAO (a non-polar solvent) caused softening of PEEK as in the case of lubrication with water (a polar solvent). The hardness of outside of the wear 


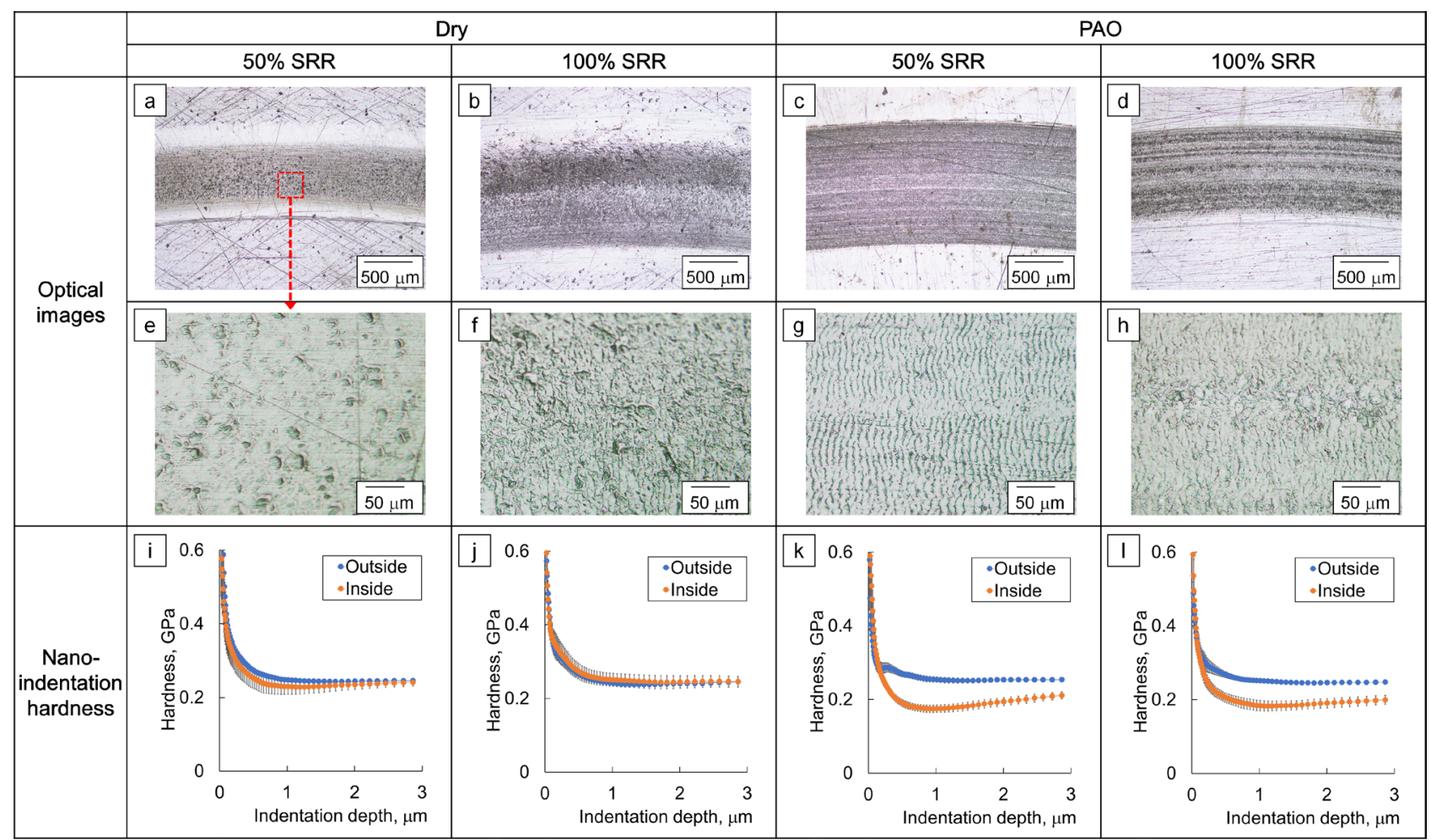

Fig. 4 (a-h) Optical images and (i-l) nanoindentation hardness of after-test PEEK plates

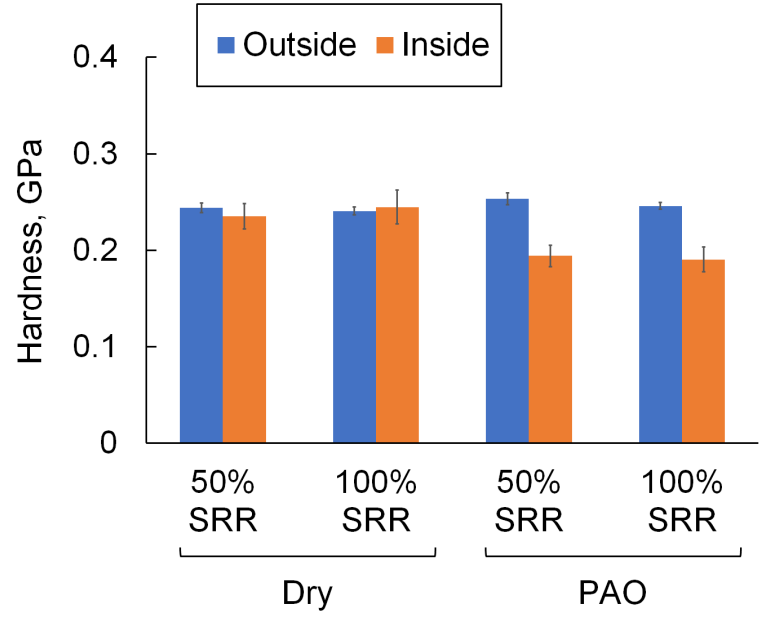

Fig. 5 Nanoindentation hardness of after-test PEEK plates at $2 \mu \mathrm{m}$ indentation depth

tracks showed almost the same value for all specimens. This indicates softening of PEEK did not occur just by immersing in PAO. Noticeably, the hardness of $50 \%$ and $100 \%$ SRR tests showed almost the same values in dry and lubricated conditions respectively, the opposite to wear rates. This implies softening of PEEK by lubrication with PAO was not the main cause of the high wear rate of the PEEK plate in the PAO 50\% SRR test. In fact, the PAO $100 \%$ SRR test showed the lowest wear rate in spite of the decrease in the hardness of PEEK.

\subsection{Investigations of PEEK transfer films on steel balls}

The wear tracks of the steel balls were analyzed after tribological tests for wear and PEEK transfer films. Optical images of all specimens in Fig. 6(a-d) showed that there was almost no wear on steel balls. As the hardness of the steel ball is much higher than that of PEEK plates, it was expected that wear mainly occurs on the PEEK plates. The balls used in the dry tests show the presence of PEEK wear debris outside the wear tracks, especially abundant with $100 \%$ SRR. In lubricated tests, the wear debris would be flushed out by PAO, but small amounts of wear debris were still observed as white residuals/ marks just outside of wear tracks. The Ra roughness of the wear tracks on steel balls did not show significant difference between the PAO 50\% and 100\% tests (approximately $0.35 \mu \mathrm{m}$ for both tests), while these $R a$ values are lower than that of the unused shot-blasted ball (approximately $0.5 \mu \mathrm{m}$ ). In addition, the $R a$ value of the wear track on the steel ball for the dry $50 \%$ test was approximately $0.3 \mu \mathrm{m}$.

The visual evaluation of the amount of PEEK transfer films on the wear track was found to be challenging so EPMA carbon mapping analysis was employed. EPMA is equipped with Wavelength Dispersive X-ray spectroscopy (WDX) which counts the number of $\mathrm{X}$-rays of a specific wavelength diffracted by a crystal, therefore it has higher resolution than Energy dispersive X-ray spectrometry (EDX), and is suitable for carbon mapping. EPMA carbon maps on after-test steel balls in Fig. 6(e-h) showed a clear difference between the steel ball wear tracks. The carbon amount is indicated by the colour scale on the map. For each of the two testing conditions a good correlation can be seen between the wear rates of PEEK plates (Fig. 3) and the carbon amount on the wear track of steel balls (Fig. 6(e-h)). Larger amounts of carbon distributed as a uniform layer were detected on the wear tracks of the steel balls used in the dry 50\% SRR test and PAO $100 \%$ SRR test. These testing conditions also led to low wear rates of PEEK plates. The PAO 100\% SRR PEEK plate showed the lowest wear rate despite the dry $50 \%$ steel ball wear track showing a much higher carbon amount. This was thought to be due to the combined effect of PEEK transfer film and oil 


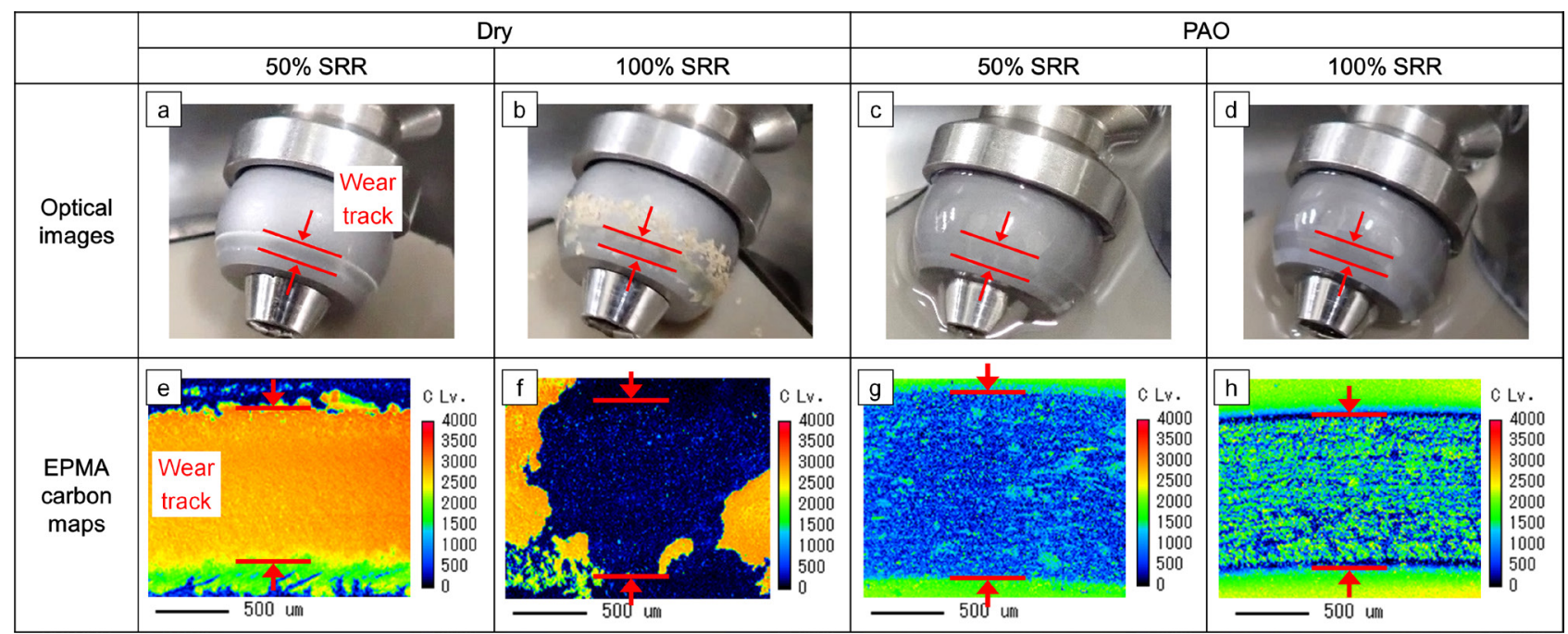

Fig. 6 (a-d) Optical images and (e-h) EPMA carbon maps of after-test steel balls

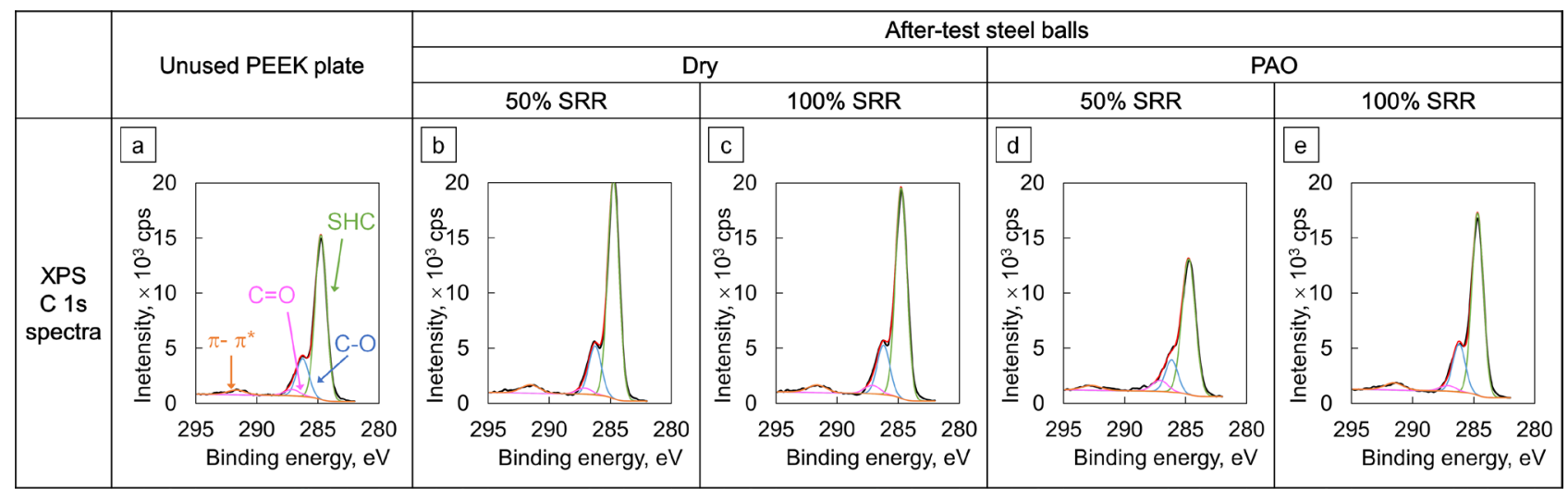

Fig. 7 XPS C 1s spectra of (a) unused PEEK plate and (b-e) after-test steel balls

lubrication which will be discussed in detail in a later section. A reduced amount of carbon was detected on the steel ball wear track from the PAO 50\% SRR test which showed a very high wear rate of the PEEK plate. The wear track of the steel ball used in the dry $100 \%$ SRR test showed non-uniform coverage with carbon varying between high and low concentrations.

The presence of carbon detected with EPMA is supposedly related to PEEK transfer films on steel ball wear tracks but this assumption is questionable in the case of PAO lubricated balls. To confirm the assumption, XPS analysis of $C$ 1s spectra was recorded on steel ball wear tracks. As Fig. 7 shows, C 1s spectra in all tests, except for the PAO 50\% SRR, showed almost identical peak curves which matched well the spectra recorded on an unused PEEK plate and the literature reported PEEK spectra [49]. A small difference in spectra is seen for the PAO 50\% SRR wear track which can be due to the very low carbon presence on the steel ball. Therefore, from the XPS results it can be concluded that EPMA carbon maps are a valid analysis of PEEK transfer films on steel ball wear tracks. Moreover, a good correlation is seen between the EPMA detected carbon amount on steel ball wear tracks and the wear rates of PEEK plates. This indicates that the presence of a PEEK transfer film on the steel ball is a key factor not just for dry conditions but also for lubricated ones.

In dry conditions, the increase in SRR from $50 \%$ to $100 \%$ mean the higher shear stresses (higher sliding speed) accelerated film removal and led to a non-uniform transfer film as seen in the dry $100 \%$ SRR test (Fig. 6(f)). Similar to the previous reports for water lubrication, PAO inhibited the formation of transfer films in the 50\% SRR test, causing direct contact between the PEEK surface and asperities of the counter steel surface. This explains the difference in surface patterns in Fig. 4. The dry $50 \%$ SRR test shows a specific pattern on the PEEK plate (Fig. 4(e)) and a thick PEEK transfer film on the steel surface which bears the hallmarks of adhesive wear or fatigue wear that can take place in the PEEK/PEEK contact. By contrast, the PAO $50 \%$ SRR test showed an abrasion pattern on PEEK (Fig. 4(g)) and a thin PEEK transfer film on the ball wear track indicating abrasive wear in the PEEK/steel contact which resulted in the most significant wear loss from all tests. The presence and thickness of PEEK transfer films on the steel counterpart are determined by the formation and removal process and thus can be significantly influenced by the operating conditions (SRR).

However, it was still unclear why the variation of SRR in the PAO lubricated tests resulted in significant differences in PEEK transfer film thicknesses. A plausible explanation could be that the SRR influences/controls the formation and removal process of PEEK transfer films and lubrication with PAO can inhibit not only the formation but also the removal of films. Therefore, additional tests were specifically devised to investigate this. 


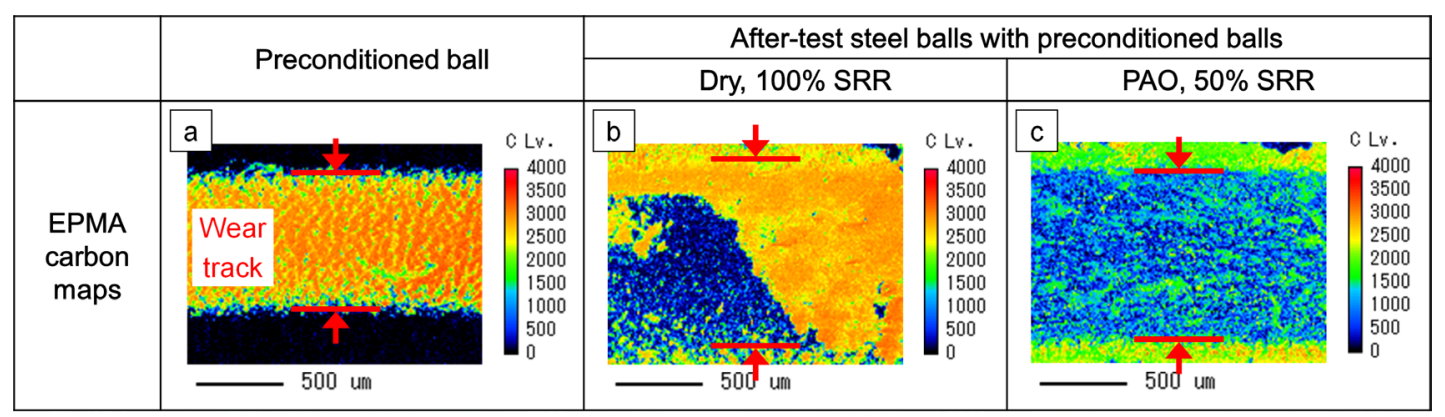

Fig. 8 EPMA carbon maps of (a) preconditioned ball and (b, c) after-test steel balls with preconditioned steel balls

\subsection{Additional tests using preconditioned steel balls}

To investigate why poorer PEEK transfer films resulted from the dry 100\% SRR and PAO 50\% SRR tests, additional testing was carried out using preconditioned steel balls. Runin dry 50\% SRR tests were carried out for 10 minutes (testing conditions identical to those used for the previous dry tests) to achieve a uniform PEEK transfer films on the steel ball wear track as seen in Fig. 6(e). The EPMA carbon map of the 10 minutes preconditioned steel ball is shown in Fig. 8(a). The preconditioned balls were then paired with unused PEEK plates and tested in dry 100\% SRR and PAO 50\% SRR for 60 minutes. The friction and wear results of these tests are shown in Figs. 9 and 10 and compared with the results obtained with new balls. The friction coefficients with the preconditioned ball and new

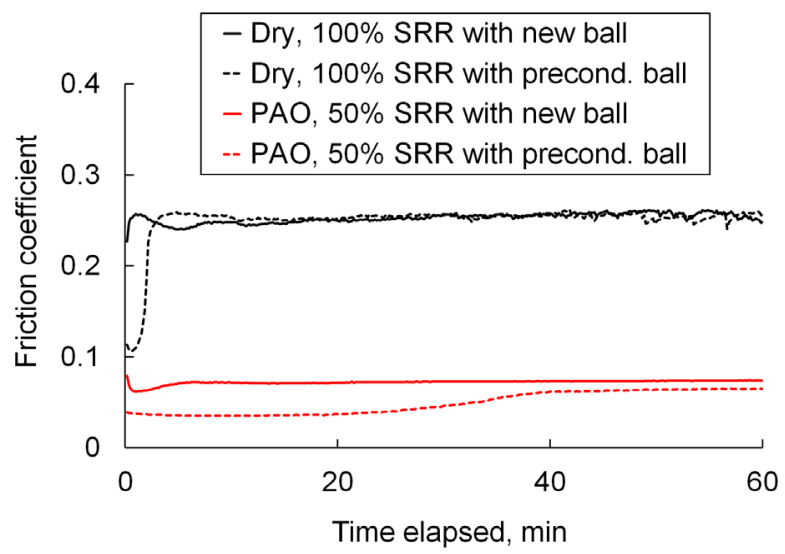

Fig. 9 Friction coefficient as a function of test time

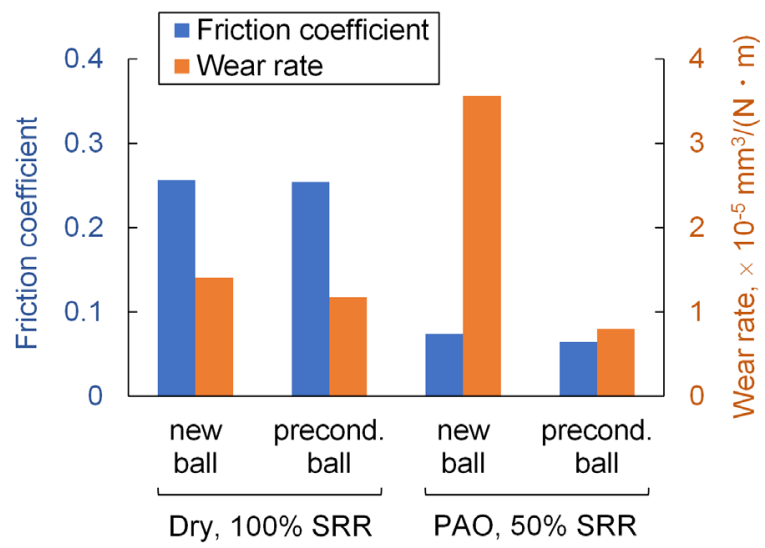

Fig. 10 Friction coefficient averaged during the last ten minutes and wear rates of PEEK plates disc started from lower values than those with the new ball and new disc but in a short time (less than 10 minutes) converged to the same level as the wear track on the PEEK disc started to form. The EPMA carbon map of the transfer film in Fig. 8(b) showed a non-uniform coverage of the wear track similar to the test with the new ball in Fig. 6(e). These results indicate that the formation and removal of PEEK transfer films in dry $100 \%$ SRR tests were very fast and the preconditioned ball did not have a meaningful effect. By contrast, the preconditioning of the ball significantly reduced friction and wear in PAO 50\% SRR test as shown in Figs. 9 and 10. Friction coefficient values with the preconditioned ball were half those with the new ball for 20 minutes and stabilized at a lower level for the rest of the test. Moreover, the wear rate of the PEEK plate decreased drastically. The transfer film with preconditioning, Fig. 8(c), was slightly thicker than that with the new ball, Fig. 6(g). These results indicate that lubrication with PAO suppressed the speed of the removal of PEEK films, and thus the initial film formed through preconditioning the balls was reasonably stable during the test.

Results from the additional tests confirmed the following assumptions: (i) SRR affects the balance of formation and removal of PEEK films and (ii) lubrication with PAO inhibits both the formation and the removal of PEEK transfer films from the steel counterparts.

\subsection{Mechanism of lubrication}

Similar to the previously reported research on water lubrication, the softening of PEEK was observed in this study, but no correlation was seen with wear behavior. On the other hand, the transfer film on the steel counterpart was found to be the dominant factor in this study and a schematic of the assumed mechanism of lubrication is presented in Fig. 11. Lubrication with PAO inhibits both the formation and the removal of PEEK transfer films, and the balance between these processes is controlled by SRR. A further similarity to water lubrication is that PAO inhibits the formation of a PEEK transfer film on the steel counter surface, thus causing direct contact between PEEK and steel. The excessive wear rate in the PAO $50 \%$ SRR test was the result of abrasive wear, inducing an abrasion pattern on the PEEK plate (Fig. 4(g)). With a higher SRR, the speeds of the formation and removal processes are both accelerated, and their balance significantly changes in the PAO 100\% SRR test. The results using preconditioned steel balls indicate that lubrication with PAO effectively suppresses the removal of PEEK transfer films once they formed, and therefore the film formation overtakes the removal in the PAO 100\% SRR test. Additionally, the combination of the PEEK transfer film and the oil film contributes to mitigating the direct contact of surfaces, showing the lowest friction coefficient and PEEK wear rate. Previous studies which investigated dry testing of PEEK 


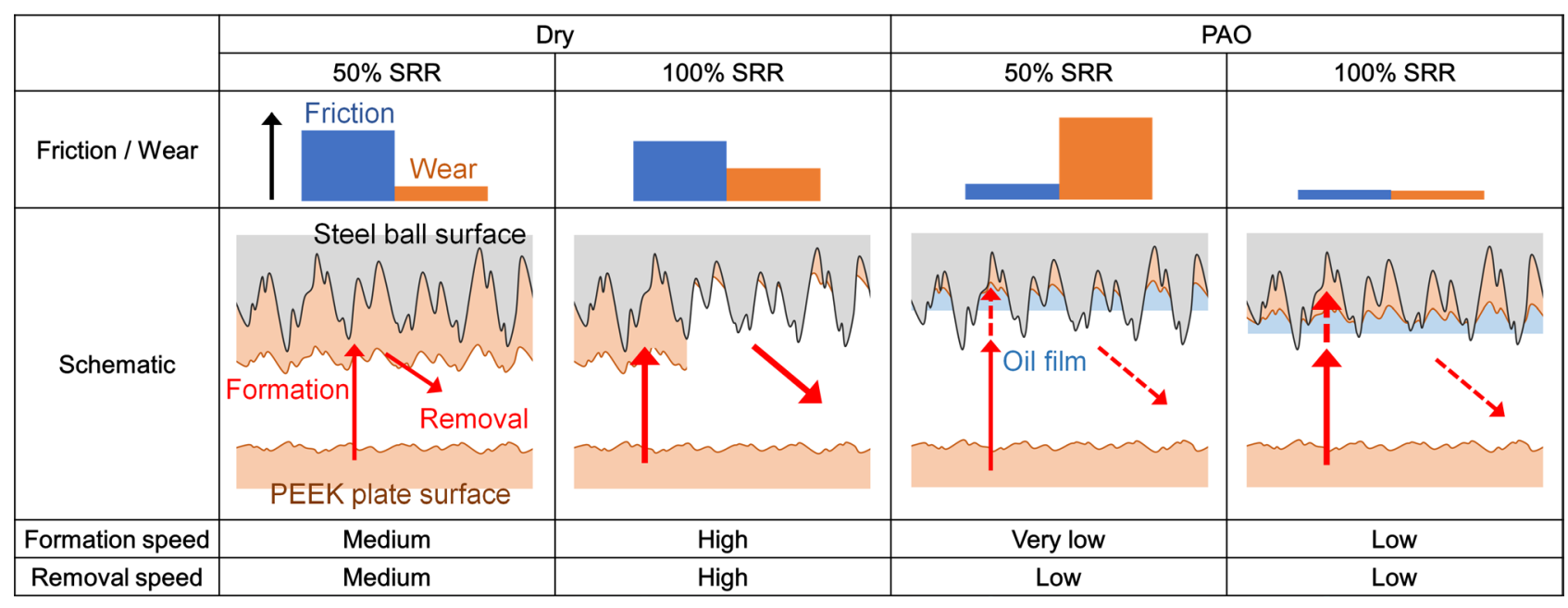

Fig. 11 Schematic of assumed mechanism of lubrication

have also reported that tribological test conditions affected the balance of formation and removal of PEEK transfer films on steel counterparts $[19,22]$. This study brings clarification into the effects of fluid lubrication by showing the impact of fluid lubrication not only on formation but also removal of films, and its dependence on the operating conditions (SRR).

\section{Conclusions}

The mechanism of lubrication of the PEEK/steel contact with a PAO base oil has been investigated by focusing on two key factors: the PEEK transfer films on the steel counterparts and the changes in hardness (softening) of PEEK specimens. The following conclusions have been drawn:

(1) Lubrication with PAO reduced the friction of the PEEK/steel contact regardless of the operating conditions. By contrast, the lubrication effects (positive or negative) on the wear of PEEK depended on SRR;

(2) Lubrication with PAO had a softening effect on the wear track of PEEK, but no correlation was established with the wear rates of PEEK;

(3) The thickness of PEEK transfer films on the steel counter surface was the main parameter that controlled PEEK wear rates;

(4) Lubrication with PAO inhibited not only the formation but also the removal of the PEEK transfer films. This explains why lubrication can play both a positive or negative role on the wear of PEEK depending on the operating conditions.

This study elucidated the fluid lubrication mechanism of PEEK and established the reason why fluid lubrication showed positive or negative effects depending on the tribological conditions. This knowledge is fundamental for the design of efficient tribological systems and the formulation of suitable lubricants for PEEK/steel applications.

\section{Acknowledgements}

The authors thank JXTG Nippon Oil \& Energy corporation, Japan for sponsoring this research study, Mr. Shinji Hasegawa from JXTG for performing the nanoindentation measurements and the Analytical Technology group from R\&D Solution Center JXTG for the EPMA/XPS analyses.

\section{References}

[1] Friedrich, K., Lu, Z. and Hager, A. M., "Recent Advances in Polymer Composites' Tribology," Wear, 190, 2, 1995, 139-144.

[2] Takeichi, Y., "Tribology of Polymer Materials," The Journal of the Surface Finishing Society of Japan, 65, 12, 2014, 562-567 (in Japanese).

[3] Myshkin, N. K., Pesetskii, S. S. and Grigoriev, A. Y., "Polymer Tribology: Current State and Applications," Tribology in Industry, 37, 3, 2015, 284-290.

[4] Theiler, G. and Gradt, T., "Friction and Wear Behaviour of Graphite Filled Polymer Composites in Hydrogen Environment," Tribology Online, 10, 2, 2015, 207-212.

[5] Sawae, Y., "Biotribology in Resin Material for Artificial Joint," Journal of Japanese Society of Tribologists, 62, 7, 2017, 435-441 (in Japanese).

[6] Nishitani, Y., Kajiyama, T. and Yamanaka, T., “Effect of Silane Coupling Agent on Tribological Properties of Hemp FiberReinforced Plant-Derived Polyamide 1010 Biomass Composites," Materials, 10, 9, 2017, 1040-1060.

[7] Gandhi, R., Jayawant, A., Bhalerao, A. and Dandagwhal, R., "Applicability of Composite Polymer Gear in Low RPM Applications - A Review," International Journal of Engineering Science Invention, 7, 4, 2018, 36-41.

[8] Friedrich, K., "Polymer Composites for Tribological Applications," Advanced Industrial and Engineering Polymer Research, 1, 1, 2018, 3-39.

[9] Kurdi, A. and Chang, L., "Recent Advances in High Performance Polymers-Tribological Aspects," Lubricants, 7, 1, 2019, 2.

[10] Walton, D. and Shi, Y. W., "A Comparison of Ratings for Plastic Gears," Proceedings of the Institution of Mechanical Engineers, Part C: Journal of Mechanical Engineering Science, 203, 1, 1989, 31-38.

[11] Li, W., Wood, A., Weidig, R. and Mao, K., "An Investigation on the Wear Behaviour of Dissimilar Polymer Gear Engagements," Wear, 271, 9-10, 2011, 2176-2183.

[12] Mertens, A. J. and Senthilvelan, S., "Durability Enhancement of Polymer Gear Using Compressed Air Cooling," Proceedings of the Institution of Mechanical Engineers, Part L: Journal of Materials: Design and Applications, 230, 2, 2016, 515-525.

[13] Singh, A. K., Siddhartha and Singh, P. K., "Polymer Spur Gears Behaviors under Different Loading Conditions: A Review," Proceedings of the Institution of Mechanical Engineers, Part J: Journal of Engineering Tribology, 232, 2, 2018, 210-228. 
[14] Mens, J. W. M. and de Gee, A. W. J., "Friction and Wear Behaviour of 18 Polymers in Contact with Steel in Environments of Air and Water," Wear, 149, 1-2, 1991, 255-268.

[15] Schelling, A., Kausch, H. H. and Roulin, A. C., "Friction Behaviour of Polyetheretherketone under Dry Reciprocating Movement," Wear, 151, 1, 1991, 129-142.

[16] Stolarski, T. A., “Tribology of Polyetheretherketone," Wear, 158, 1-2, 1992, 71-78.

[17] Lu, Z. P. and Friedrich, K., "On Sliding Friction and Wear of PEEK and Its Composites," Wear, 181-183, Part 2, 1995, 624-631.

[18] Koike, H., Kida, K., Santos, E. C., Rozwadowska, J., Kashima, Y. and Kanemasu, K., "Self-Lubrication of PEEK Polymer Bearings in Rolling Contact Fatigue under Radial Loads," Tribology International, 49, 2012, 30-38.

[19] Laux, K. A. and Schwartz, C. J., "Influence of Linear Reciprocating and Multi-Directional Sliding on PEEK Wear Performance and Transfer Film Formation," Wear, 301, 1-2, 2013, 727-734.

[20] Laux, K. A., Jean-Fulcrand, A., Sue, H. J., Bremner, T. and Wong, J. S. S., "The Influence of Surface Properties on Sliding Contact Temperature and Friction for Polyetheretherketone (PEEK)," Polymer, 103, 2016, 397-404.

[21] Zalaznik, M., Kalin, M. and Novak, S., "Influence of the Processing Temperature on the Tribological and Mechanical Properties of PolyEther-Ether-Ketone (PEEK) Polymer," Tribology International, 94, 2016, 92-97.

[22] Bahadur, S., "The Development of Transfer Layers and Their Role in Polymer Tribology," Wear, 245, 1-2, 2000, 92-99.

[23] Puhan, D. and Wong, J. S. S., "Properties of Polyetheretherketone (PEEK) Transferred Materials in a PEEK-Steel Contact," Tribology International, 135, 2019, 189-199.

[24] Kalin, M., Zalaznik, M. and Novak, S., "Wear and Friction Behaviour of Poly-Ether-Ether-Ketone (PEEK) Filled with Graphene, $\mathrm{WS}_{2}$ and CNT Nanoparticles," Wear, 332-333, 2015, 855-862.

[25] Minami, I., Kubo, T., Nanao, H., Mori, S., Iwata, H. and Fujita, M., "Surface Chemistry for Improvement in Load-Carrying Capacity of Poly(Ether-Ether-Ketone)-Based Materials by Poly(Tetrafluoroethylene)," Tribology Online, 3, 3, 2008, 190-194.

[26] Pei, X. Q., Bennewitz, R. and Schlarb, A. K., "Mechanisms of Friction and Wear Reduction by Carbon Fiber Reinforcement of PEEK," Tribology Letters, 58, 3, 2015, 42.

[27] Rodriguez, V., Sukumaran, J., Schlarb, A. K. and De Baets, P., "Influence of Solid Lubricants on Tribological Properties of Polyetheretherketone (PEEK)," Tribology International, 103, 2016, 45-57.

[28] Zalaznik, M., Kalin, M., Novak, S. and Jakša, G., “Effect of the Type, Size and Concentration of Solid Lubricants on the Tribological Properties of the Polymer PEEK," Wear, 364-365, 2016, 31-39.

[29] Kumar, D., Rajmohan, T. and Venkatachalapathi, S., "Wear Behavior of PEEK Matrix Composites: A Review," Materials Today: Proceedings, 5, 6, 2018, 14583-14589.

[30] Zhang, G., Wetzel, B. and Wang, Q., “Tribological Behavior of PEEK-Based Materials under Mixed and Boundary Lubrication Conditions," Tribology International, 88, 2015, 153-161.

[31] Sumer, M., Unal, H. and Mimaroglu, A., "Evaluation of Tribological Behaviour of PEEK and Glass Fibre Reinforced PEEK Composite under Dry Sliding and Water Lubricated Conditions," Wear, 265,
7-8, 2008, 1061-1065.

[32] Gao, C., Zhang, G., Wang, T. and Wang, Q., “Enhancing the Tribological Performance of PEEK Exposed to Water-Lubrication by Filling Goethite ( $\alpha$-FeOOH) Nanoparticles," RSC Advances, 6, 56, 2016, 51247-51256.

[33] Kurdi, A., Wang, H. and Chang, L., "Effect of Nano-Sized $\mathrm{TiO}_{2}$ Addition on Tribological Behaviour of Poly Ether Ether Ketone Composite," Tribology International, 117, 2018, 225-235.

[34] Yamamoto, Y. and Takashima, T., "Friction and Wear of Water Lubricated PEEK and PPS Sliding Contacts," Wear, 253, 7-8, 2002, 820-826.

[35] Yamaguchi, T. and Hokkirigawa, K., "Friction and Wear Properties of Peek Resin Filled with RB Ceramics Particles under Water Lubricated Condition," Tribology Online, 11, 6, 2016, 653-660.

[36] Briscoe, B. J., Stolarski, T. A. and Davies, G. J., "Boundary Lubrication of Thermoplastic Polymers in Model Fluids," Tribology International, 17, 3, 1984, 129-137.

[37] Akagaki, T. and Kawabata, M., "Effects of Counterface Surface Roughness on Friction and Wear of PEEK Materials under OilLubricated Conditions," Tribology Online, 11, 3, 2016, 494-502.

[38] Akagaki, T., Kataoka, K. and Kawabata, M., "Wear Debris Analysis of Seizure Behaviors of PEEK Materials in Oil," Tribology Online, $11,5,2016,623-631$.

[39] Li, X. and Bhushan, B., "A Review of Nanoindentation Continuous Stiffness Measurement Technique and Its Applications," Materials Characterization, 48, 1, 2002, 11-36.

[40] Iqbal, T., Briscoe, B. J. and Luckham, P. F., "Surface Plasticization of Poly(Ether Ether Ketone)," European Polymer Journal, 47, 12, 2011, 2244-2258.

[41] Voyiadjis, G. Z., Samadi-Dooki, A. and Malekmotiei, L., "Nanoindentation of High Performance Semicrystalline Polymers: A Case Study on PEEK," Polymer Testing, 61, 2017, 57-64.

[42] Hantsche, H., "Comparison of Basic Principles of the Surfacespecific Analytical Methods: AES/SAM, ESCA (XPS), SIMS, and ISS with X-ray Microanalysis, and Some Applications in Research and Industry," Scanning, 11, 6, 1989, 257-280.

[43] Hamrock, B. J. and Dowson, D., "Isothermal Elastohydrodynamic Lubrication of Point Contacts III - Fully Flooded Results," NASA Technical Note, 1976, D-8317.

[44] Hamrock, B. J. and Dowson, D., “Elastohydrodynamic Lubrication of Elliptical Contacts for Materials of Low Elastic Modulus. 1-Fully Flooded Conjunction.," NASA Technical Note, 1977, D-8528.

[45] Marx, N., Guegan, J. and Spikes, H. A., "Elastohydrodynamic Film Thickness of Soft EHL Contacts Using Optical Interferometry," Tribology International, 99, 2016, 267-277.

[46] Schallamach, A., "Friction and Abrasion of Rubber," Wear, 1, 5, 1958, 384-417.

[47] Bonne, M., Briscoe, B. J., Lawrence, C. J., Manimaaran, S., Parsonage, D. and Allan, A., "Nano-Indentation of Scratched Poly(Methyl Methacrylate) Surfaces," Tribology Letters, 18, 2, 2005, 125-133.

[48] Miyake, K., Motoda, T. and Sasaki, S., "Nanoindentation," Journal of Japanese Society of Tribologists, 51, 7, 2006, 518-523 (in Japanese).

[49] Li, T. Q., Zhang, M. Q., Song, L. and Zeng, H. M., "Friction Induced Mechanochemical and Mechanophysical Changes in High Performance Semicrystalline Polymer," Polymer, 40, 16, 1999, 44514458 